\title{
Properties and Characterization of ALD Grown Dielectric Oxides for MIS Structures
}

\author{
S. Gieraztowska ${ }^{a * *}$, D. Sztenkiel ${ }^{a}$, E. Guziewicz ${ }^{a}$, M. Godlewski $^{a, b}$, G. Euka $^{a}$, \\ B.S. Witkowski ${ }^{a}$, Ł. WAChNicki $^{a}$, E. ŁusAkowska $^{a}$, T. Dietl $^{a, c}$ AND M. SAWicki ${ }^{a}$ \\ ${ }^{a}$ Institute of Physics, Polish Academy of Sciences, al. Lotników 32/46, 02-668 Warszawa, Poland \\ ${ }^{b}$ Natural Sciences College of Science, Cardinal S. Wyszyński University, Warszawa, Poland \\ ${ }^{c}$ Institute of Theoretical Physics, University of Warsaw, Warszawa, Poland
}

\begin{abstract}
We report on an extensive structural and electrical characterization of undergate dielectric oxide insulators $\mathrm{Al}_{2} \mathrm{O}_{3}$ and $\mathrm{HfO}_{2}$ grown by atomic layer deposition. We elaborate the atomic layer deposition growth window for these oxides, finding that the 40-100 $\mathrm{nm}$ thick layers of both oxides exhibit fine surface flatness and required amorphous structure. These layers constitute a base for further metallic gate evaporation to complete the metal-insulator-semiconductor structure. Our best devices survive energizing up to $\approx 3 \mathrm{MV} / \mathrm{cm}$ at $77 \mathrm{~K}$ with the leakage current staying below the state-of-the-art level of $1 \mathrm{nA}$. At these conditions the displaced charge corresponds to a change of the sheet carrier density of $3 \times 10^{13} \mathrm{~cm}^{-2}$, which promises an effective modulation of the micromagnetic properties in diluted ferromagnetic semiconductors.
\end{abstract}

PACS: 81.15.Gh, 77.55.-g, 77.84.Bw, 81.05.Ea

\section{Introduction}

One of the main aspects in the spintronic research is the development of diluted ferromagnetic semiconductors (DFS) and their use as a test bed for concept and device proofs for future applications. Ferromagnetism of these compounds is brought about by the exchange interaction among magnetic impurity spins (so far successful only for Mn) and valence band holes. Accordingly, ferromagnetism of these materials depends on the hole concentration $p$ and, thus, can be controlled by the gate electric field in metal-insulator-semiconductor (MIS) structures with the magnetically doped channel. After the first successful realization of this concept [1], a greater technological progress has been achieved when high- $\kappa$ dielectric oxides were employed. Except of high dielectric constants these new gate insulators offer large breakdown fields and oxides like zirconium dioxide $\left(\mathrm{ZrO}_{2}, \kappa=20-23\right)$, hafnium dioxide $\left(\mathrm{HfO}_{2}, \kappa=16-19\right)$ and aluminium oxide $\left(\mathrm{Al}_{2} \mathrm{O}_{3}\right.$, $\kappa=8-9)$ [2] are seen as the alternatives to $\mathrm{SiO}_{2}$ in commercial electronics, as $\mathrm{HfO}_{2}$ has already been used in $45 \mathrm{~nm}$ node processors [3]. On the ground of spintronic research these oxides allow us now to modulate not only the Curie temperature $T_{\mathrm{C}}$, but all the micromagnetic characteristics in an unprecedented wide range $[4,5]$.

These materials are grown by the atomic layer deposition (ALD) method [6], which is a a self-limiting and sequential growth process, which enables using very reactive precursors. This method in general offers high conformability (films of uniform thickness can be ob-

\footnotetext{
* corresponding author; e-mail: sgieral@ifpan.edu.pl
}

tained on substrates with complex shape [7]), smooth surfaces, a precise control of composition and thickness, which together with excellent patterning properties up to nanometer scale, makes it ideal solution for various undergate dielectrics deposition. Simultaneously, all of this can be obtained at relatively low growth temperatures, reaching even below $100^{\circ} \mathrm{C}$, as recently shown for $\mathrm{ZnO}$ [8]. This in turn makes this method ideal for low temperature budget applications like hybrid organic/inorganic and 3D electronics [9-11] or in connection with DFS like (Ga,Mn) As [12, 13].

\section{Experimental}

The high- $\kappa$ thin films $\mathrm{HfO}_{2}$ and $\mathrm{Al}_{2} \mathrm{O}_{3}$ were deposited on glass, $n$-Si, $p$-GaAs and $(\mathrm{Ga}, \mathrm{Mn}) \mathrm{As}$ substrates by the ALD method in a Savannah-100 reactor from Cambridge NanoTech Company. We used deionized water as an oxygen precursor, tetrakis(dimethylamido)hafnium (TDMAH) as a hafnium precursor and trimethylaluminum (TMA) as an aluminum precursor. The dielectric oxides were obtained at low temperature (between $80^{\circ} \mathrm{C}$ and $240^{\circ} \mathrm{C}$ ) by double-exchange chemical reactions

$$
\begin{aligned}
& \mathrm{Hf}\left[\left(\mathrm{CH}_{3}\right)_{2} \mathrm{~N}\right]_{4}+2 \mathrm{H}_{2} \mathrm{O} \rightarrow \mathrm{HfO}_{2}+4 \mathrm{HN}\left(\mathrm{CH}_{3}\right)_{2}, \\
& 2 \mathrm{Al}\left(\mathrm{CH}_{3}\right)_{3}+3 \mathrm{H}_{2} \mathrm{O} \rightarrow \mathrm{Al}_{2} \mathrm{O}_{3}+6 \mathrm{CH}_{4} .
\end{aligned}
$$

The structural characterization was carried out by the $\mathrm{X}$-ray diffraction (XRD) method. The surface morphology and cross-section of the films were investigated by atomic force microscopy (AFM) and scanning electron microscopy (SEM). To obtain capacitor-like structures for gating, a $3 \mathrm{~nm} \mathrm{Cr} / 50 \mathrm{~nm}$ Au gate electrode was evaporated on the hand-painted photoresist mask. After the lift-off the final electrical connections to the $\mathrm{Au}$ gate and 
base semiconductor were done by silver-based conductive apoxy and indium, respectively. In order to put our gating technology to the ultimate test, the area of our capacitors was about $2 \times 2 \mathrm{~mm}^{2}$.

\section{Results and discussion}

In order to increase efficiency and yield of the gated structures we started our project from elaboration of the basic growth conditions that may influence the electrical properties of gate dielectrics. For this purpose we used $n$-Si substrates. We began with the growth temperature $\left(T_{\mathrm{g}}\right)$ as it is one of the most important parameter in the ALD process, as it controls the surface coverage. We started from elaborating the ALD growth window, which is the temperature range where the growth rate is constant and the growth process is the closest to one monolayer per cycle. We established that the ALD window for $\mathrm{HfO}_{2}$ is in the range from $130{ }^{\circ} \mathrm{C}$ to $140{ }^{\circ} \mathrm{C}$ giving the growth rate of $1.4 \AA$ per cycle (Fig. 1a) and from $180{ }^{\circ} \mathrm{C}$ to $200{ }^{\circ} \mathrm{C}$ for $\mathrm{Al}_{2} \mathrm{O}_{3}$ giving the growth rate of $1.0 \AA$ per cycle (Fig. 1b).

The XRD measurements reveal that the crystallographic structure depends on the thickness of the layer. Although $\mathrm{Al}_{2} \mathrm{O}_{3}$ grown at the ALD window conditions $\left(T_{\mathrm{g}}=200^{\circ} \mathrm{C}\right.$, Fig. 2a) shows consistently amorphous structure for the wide range of the thickness of the layers, the crystallographic structure of $\mathrm{HfO}_{2}\left(T_{\mathrm{g}}=135^{\circ} \mathrm{C}\right.$, Fig. 2b) changes from the amorphous for the lower thick- nesses to a homogeneously nanocrystalline one for the very thick layers. We note that films with a thickness of above about $200 \mathrm{~nm}$ show a tendency to crystallize even at low process temperature. These layers crystallize in monoclinic structure. Since the amorphous structure is expected to have substantially reduced leakage current, usually associated with presence of the grain boundaries of highly crystalline films [14], this is the amorphous structure that is preferred for the gate applications. Therefore, the crystallographic dependence on the thickness of the $\mathrm{HfO}_{2}$ layers instructs us that we should work with rather thin $\mathrm{HfO}_{2}$ layers in order to increase technological yield of manufactured gated structures.

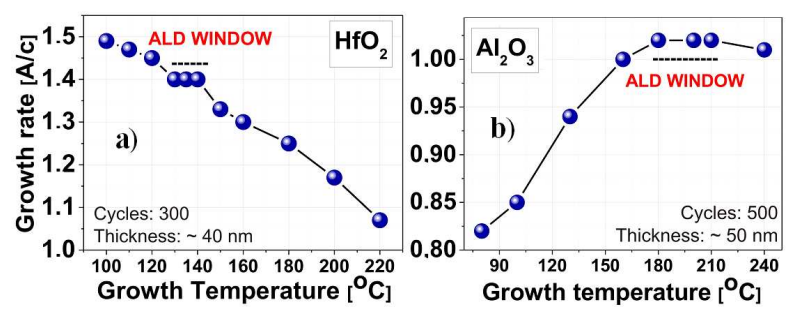

Fig. 1. The growth rate of the (a) $\mathrm{HfO}_{2}$ and (b) $\mathrm{Al}_{2} \mathrm{O}_{3}$ films as a function of deposition temperature. ALD window is in the range of temperatures: (a) $130-140{ }^{\circ} \mathrm{C}$ and (b) $180-200{ }^{\circ} \mathrm{C}$.
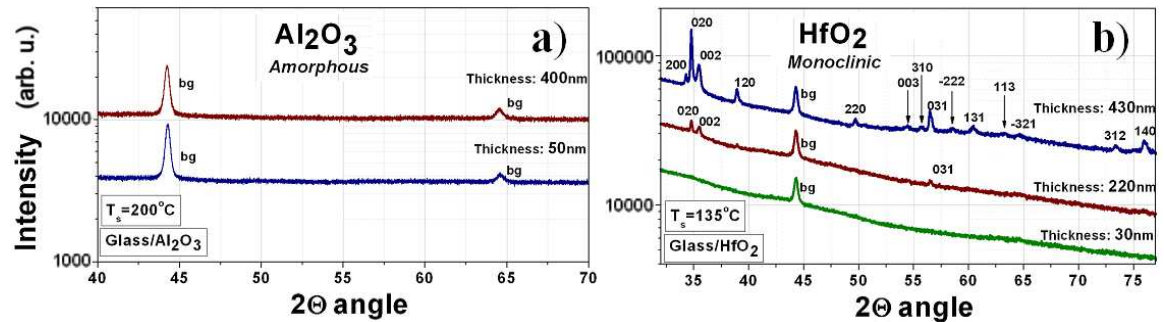

Fig. 2. The XRD spectra of (a) $\mathrm{HfO}_{2}$ films grown at temperature of $200{ }^{\circ} \mathrm{C}$, and (b) $\mathrm{Al}_{2} \mathrm{O}_{3}$ films grown at temperature of $135^{\circ} \mathrm{C}$ by the ALD on glass substrates.

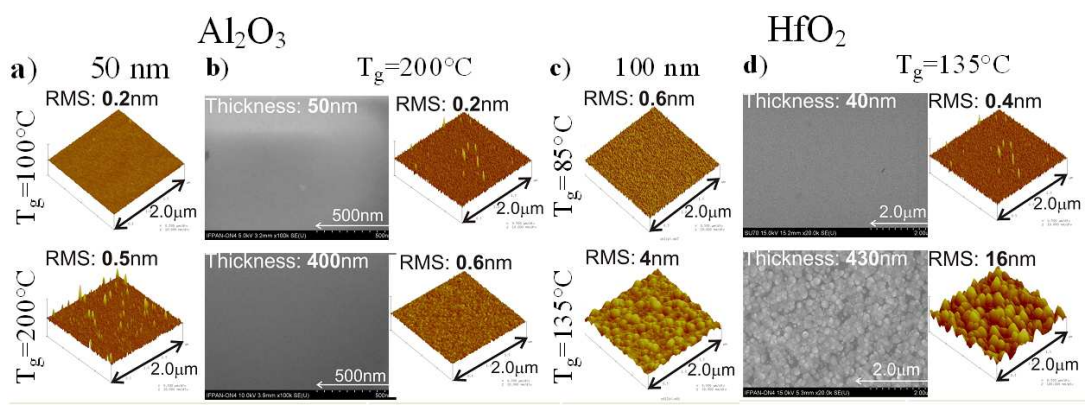

Fig. 3. SEM and AFM surface morphology studies of $\mathrm{Al}_{2} \mathrm{O}_{3}$ (a), (b) and $\mathrm{HfO}_{2}$ (c), (d) thin films on Si substrate.

The leakage current, and so the gate efficiency, depends also on the surface roughness, which should be kept at the lowest possible value. We assess this parameter by AFM studies, finding that the root mean square (RMS) 
value of the surface roughness depends both on $T_{\mathrm{g}}$ and the layer thickness. For the medium- $\kappa \mathrm{Al}_{2} \mathrm{O}_{3}$ the RMS decreases about twice when we reduce $T_{\mathrm{g}}$ or layer thickness (see Fig. 3a and b). All these values (about $0.5 \mathrm{~nm}$ ) are regarded as very low indicating that for gating application this oxide can be grown at its growth window conditions. The situation is completely different for $\mathrm{HfO}_{2}$, because the layer grown at growth window conditions exhibits prohibitively high values of surface RMS, 4-16 nm (see Fig. 3d). However, we find that RMS improves substantially with reducing both $T_{\mathrm{g}}$ and the layer thickness, reaching an acceptable value of $0.6 \mathrm{~nm}$ for $T_{\mathrm{g}}=85^{\circ} \mathrm{C}$ and thickness of $100 \mathrm{~nm}$ (Fig. 3c). We select these conditions as the basic growth parameters for deposition of gate insulating layers.

The ability of electric field control of magnetic anisotropy, as recently demonstrated in (Ga,Mn)As [3], has became recently a subject of intensive studies as it leads to all-electric magnetization reversal. Gated DFS or diluted magnetic semiconductors in general offer also a deep insight into the most fundamental questions of the nature of magnetic coupling in magnetically diluted systems. Therefore we concentrate our efforts on (Ga,Mn)As layers as our semiconductor channels of the prime interest. Having magnetic measurements in mind (where the experimental abilities are determined by the sheer number of spins, which for a given thickness depends on the area of the structure), but also to put our structures to the ultimate tests, we make our capacitors of a large area, typically of about $4 \mathrm{~mm}^{2}$. The general layout of the capacitor MIS structure is presented in the inset to Fig. 4, whereas the typical cross-sectional SEM image constitute the main body of the figure. We find that both interfaces: semiconductor/dielectric-oxide, and the oxide/metallic-gate are fine and smooth. Moreover, a multiple SEM imaging (not shown) confirms the same structure morphology along the millimeters long sections of our devices.

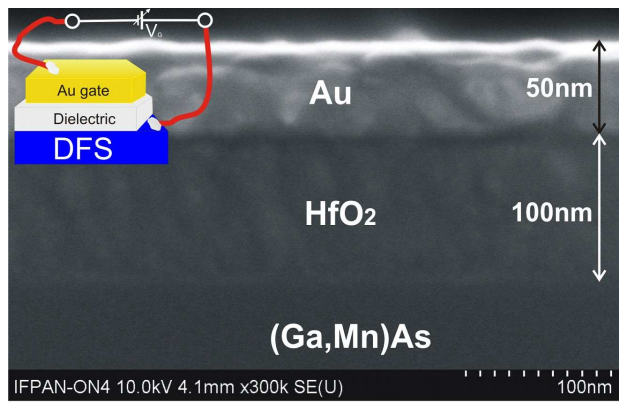

Fig. 4. The cross-section SEM image of the real test structure produced from $\mathrm{Au}$ gate $(50 \mathrm{~nm})$ and $\mathrm{HfO}_{2}$ high- $\kappa$ dielectric $(100 \mathrm{~nm})$ grown at $85^{\circ} \mathrm{C}$ on GaMnAs substrate. The inset shows the general layout of our capacitor structure.

We usually define up to 12 individual capacitors in one process, which are tested for the break-down electric field in a home made $I-V$ tester. Figure 5 presents

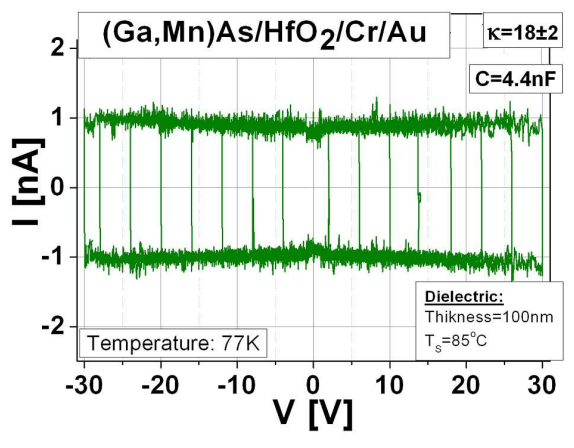

Fig. 5. The $I-V$ characteristic for the (Ga,Mn)As/100 nm $\mathrm{HfO}_{2} /(3 \mathrm{~nm} \mathrm{Cr}+50 \mathrm{~nm} \mathrm{Au})$ capacitor recorded at $77 \mathrm{~K}$. The voltage across the structure was cyclically ramped to progressively higher voltage in $4 \mathrm{~V}$ increments. The test was stopped at $30 \mathrm{~V}$ which corresponded to electric field of $3 \mathrm{MV} / \mathrm{cm}$.

$I-V$ dependence for one of the best structures obtained so far on $(\mathrm{Ga}, \mathrm{Mn}) \mathrm{As}$. Horizontalness of the $I-V$ curve indicates that the leakage current stays below $0.1 \mathrm{nA}$ for this device (or $3 \times 10^{-9} \mathrm{~A} / \mathrm{cm}^{2}$ in absolute units) up to electric field of $3 \mathrm{MV} / \mathrm{cm}$. Although the latter number does not compare very favorably to the best numbers reported in the literature $[2,12,13]$, the leakage current limit puts our structures among the best contemporary state-of-the-art devices. Direct measurements of the capacitances of our structures gives dielectric constants of $\mathrm{HfO}_{2} \kappa \approx 18 \pm 2$. A typical device yield for parameters given in Fig. 4 exceeds $50 \%$.

The values presented here allow to gate in or out of the base semiconductor a charge that corresponds to surface density up to about $\Delta p_{s}=3 \times 10^{13} \mathrm{~cm}^{-2}$. This value already promises an effective modulation of the micromagnetic properties in DFS-based structures.

\section{Conclusions}

High quality films of high- $k$ dielectric oxides are deposited at low temperature. Growth parameters are optimized for the monolayer growth mode and for maximum smoothness required for gating.

First working MIS structures based on (Ga,Mn)As are successfully fabricated exhibiting the state-of-the-art electrical parameters, offering an ability to effective gating of the magnetic properties of this DFS compound.

\section{Acknowledgments}

The authors are indebted to J. Sadowski for proving (Ga,Mn)As layers for testing purposes. The work was supported in part by the European Research Council through the FunDMS Advanced Grant within the "Ideas" 7th Framework Programme of the EC, EC Network SemiSpinNet (PITN-GA-2008-215368), and InTechFun (POIG.01.03.01-00-159/08) from EU. 


\section{References}

[1] H. Ohno, D. Chiba, F. Matsukura, T. Omiya, E. Abe, T. Dietl, Y. Ohno, K. Ohtani, Nature 408, 944 (2000).

[2] M. J. Biercuk, D.J. Monsma, C.M. Marcus, J.S. Becker, R.G. Gordon, Appl. Phys. Lett. 83, 2405 (2003).

[3] C. Zhao, T. Witters, P. Breimer, J. Maes, M. Caymax, S. De Gendt, Microelectron. Eng. 84, 7 (2007).

[4] D. Chiba, F. Matsukura, H. Ohno, Appl. Phys. Lett. 89, 162505 (2006).

[5] M. Sawicki, D. Chiba, A. Korbecka, Y. Nishitani, F. Matsukura, J.A. Majewski, T. Dietl, H. Ohno, $\mathrm{Na}$ ture Phys. 6, 22 (2010).

[6] T. Suntola, J. Antson, US Patent 4058430 (1977).

[7] O. Sneh, R.B. Clark-Phelps, A.R. Londergan, J. Winkler, T.E. Seidel, Thin Solid Films 402, 248 (2002).

[8] E. Guziewicz, I.A. Kowalik, M. Godlewski, K. Kopałko, V. Osinniy, A. Wójcik, S. Yatsunenko, E. Łusakowska, W. Paszkowicz, M. Guziewicz, J. Appl. Phys. 103, 033515 (2008).
[9] N. Huby, G. Tallarida, M. Kutrzeba, S. Ferrari, E. Guziewicz, L. Wachnicki, M. Godlewski, Microelectron. Eng. 85, 2442 (2008).

[10] G. Luka, T. Krajewski, L. Wachnicki, A. Szczepanik, J.D. Fidelus, A. Szczerbakow, E. Lusakowska, K. Kopalko, E. Guziewicz, M. Godlewski, Acta Phys. Pol. A 114, 1229 (2008)

[11] M. Godlewski, E. Guziewicz, J. Szade, A. Wojcik-Głodowska, Ł. Wachnicki, T. Krajewski, K. Kopalko, R. Jakieła, S. Yatsunenko, E. Przezdziecka, P. Kruszewski, N. Huby, G. Tallarida, S. Ferrari, Microelectron. Eng. 85, 2434 (2008).

[12] Y. Nishitani, D. Chiba, F. Matsukura, H. Ohno, J. Appl. Phys. 103, 07D139 (2008).

[13] M. Endo, D. Chiba, Y. Nishitani, F. Matsukura H. Ohno, J. Supercond. Nov. Magn. 20, 409 (2007).

[14] P. Balk, J. Non-Cryst. Solids 187, 1 (1995). 